Radiologe 2018 $\cdot 58: 192-193$

https://doi.org/10.1007/s00117-018-0366-8

(c) Springer Medizin Verlag $\mathrm{GmbH}$, ein Teil von Springer Nature 2018

CrossMark

\section{S. Delorme ${ }^{1} \cdot$ R. Loose ${ }^{2}$}

'Radiologie/E010, Deutsches Krebsforschungszentrum, Stiftung des öffentlichen Rechts, Heidelberg, Deutschland

${ }^{2}$ Institut für Radiologie und Nuklearmedizin, Klinikum Nürnberg, Nürnberg, Deutschland

\title{
Evolution auf leisen Sohlen
}

Liebe Leserinnen und Leser,

spricht man von neuen Entwicklungen in der bildgebenden Diagnostik, denkt man zunächst an die seit über 30 Jahren in der klinischen Bildgebung etablierte Magnetresonanztomographie (MRT) mit ihren immensen Möglichkeiten: Wenn man in einer aktuellen Untersuchung zum Vergleich nur 10 Jahre alte Voraufnahmen lädt, ist nicht zu übersehen, was sich allein schon in puncto Bildqualität getan hat. Hinzu kommen funktionelle Untersuchungen, von denen in nicht allzu ferner Vergangenheit nur auf Insiderkongressen die Rede war und die heute in der Routine angekommen sind. Diffusionsbildgebung ist ein Beispiel. Verglichen damit erscheinen die Entwicklungen von Methoden, die auf ionisierender Strahlung beruhen, eher moderat: Bessere Bildqualität, natürlich, aber etwas wirklich Neues? Zugegeben, die Goldgräberzeit ist vorbei, was indirekt heißt, dass hier ein so hohes $\mathrm{Maß}$ an Qualität und Zuverlässigkeit erreicht ist, dass Fortschritte langsamer kommen und weniger spektakulär sind. Sie vollziehen sich oft im Stillen, nur hier und da von einer kleinen Fanfare (Spiral-CT [Computertomographie], Mehrzeilentechnik, Dual-Energy-CT, digitale Volumendomographie [DVT] bzw. Cone-Beam-CT [CBCT], MammaTomosynthese ...) begleitet, vergleichbar der stillen Revolution im Ultraschall [6]. Führen wir uns einmal vor Augen, was beispielsweise in der Computertomographie heute selbstverständlich ist und noch vor 20 Jahren allenfalls ein Wunschtraum war:

- Schnelligkeit: eine angiographische Phase des gesamten Abdomens in wenigen Sekunden, isotrope Ganz- körperbildgebung bei Polytrauma, virtuelle Navigation durch Hohlorgane. Wir kennen noch Zeiten, da standen im "fast mode“ ganze 23 Schichten à $5 \mathrm{~s}$ für eine Kontrastmittelserie zur Verfügung, gefolgt von der Meldung ,wait for tube cooling". Vom Darm sah man, dass er da war (sicherheitshalber mit Barium gefüllt), aber mehr war darüber nicht zu sagen. Für eine Beurteilung allein der Wanddicke hätte die Peristaltik die Zeitauflösung überfordert.

- Auflösung: annähernd isotrope Daten und multiplanare Rekonstruktionen in allen Ebenen (war früher ein Privileg der MRT), gestochen scharfe EKG(Elektrokardiogramm)-getriggerte Darstellung der Koronargefäße.

- Kontrast: CT-Angiographien mit niedriger Röhrenspannung und verbessertem Jodkontrast.

- Funktionelle Bildgebung: CT- und PET(Positronenemissionstomographie)-Scanner in einem Gerät vereint.

Gleichwohl ist die Liste der Wünsche lang, und an deren Erfüllung wird gearbeitet. So ist die Computertomographie weiterhin mit über $60 \%$ in Deutschland für den größten Dosisbeitrag in der medizinischen Strahlenanwendung verantwortlich. Durch moderne iterative Rekonstruktionsverfahren, wie sie bereits seit Langem in der SPECT (,single photon emission computed tomography") und PET gebräuchlich sind, können Bildrauschen und Rekonstruktionsartefakte vermindert und so im Gegenzug der Röhrenstrom und damit die Patientenexposition reduziert werden [1]. Nun wird es Zeit, dass diese neuen Entwick- lungen auch breit in der täglichen Praxis umgesetzt werden.

Das Prinzip der Bildberechnung in der Computertomographie ist nicht an die Konstruktion eines klassischen CTScanners gebunden, sondern lässt sich auf C-Arm-Systeme mit Flachbilddetektoren übertragen. Trotz Einschränkungen, z. B. hinsichtlich der Scangeschwindigkeit, stellt so die digitale Volumentomographie bzw. Cone-Beam-CT (eigentlich ist ein modernes Mehrzeilen-SpiralCT nichts anderes) eine kostengünstige und flexible Bereicherung der konventionellen Radiographie dar [2]. Dass sie im Vergleich zur Radiographie oft eine höhere Strahlendosis verursachen und die aktuelle Vergütungsstruktur falsche Anreize setzt, steht auf einem anderen Blatt.

Mit PET/CT-Hybridgeräten ist eine anatomische Zuordnung der Traceranreicherung möglich, und zugleich dienen die Hounsfield-Werte der CT anstelle von mit Isotopenquellen erzeugten Schwächungskarten zur Schwächungskorrektur. Langsam bekommt die Blockade der gesetzlichen ambulanten Vergütung dieser potenten Methode an einzelnen Stellen Risse, so dass auch Patienten in Deutschland mehr von ihr profitieren können. Ein Beispiel für ihr Potenzial ist die Anwendung eines markierten PSMA-Liganden in der PET [3], die sich innerhalb weniger Jahre als Durchbruch in der Diagnostik des Prostatakarzinoms erwiesen hat - beim erneuten PSA-Anstieg nach primärer Behandlung ganz sicher, in Zukunft möglicherweise auch zur Zielvolumendefinition für die Strahlentherapie des Primärtumors. In zahlreichen anatomischen Strukturen ist allerdings die MRT der CT als morphologisches Bildgebungsverfahren überlegen, wie z. B. im Zentralnervensys- 
tem (ZNS), in Gelenken oder im Becken. Die PET/MRT-Hybridbildgebung ist in vielerlei Hinsicht nicht trivial, ungeachtet der Herausforderung, solche Geräte überhaupt zu bauen. So ist beispielsweise die Schwächungskorrektur kein automatischer Bestandteil wie bei der PET/CT und lässt sich auch allein aufgrund der Signalintensitäten nicht ohne Weiteres erzeugen. Gleichwohl ist das Potenzial der Methode groß, denkt man z. B. an die Aufzeichnung von Bewegung in Echtzeit und die Korrektur von Mismatches an bewegten Organen [4].

Weitere technische Entwicklungen ergeben sich unmittelbar aus der Physik, genauer gesagt, der Wechselwirkung zwischen Photonen und Materie. Eine wurde bereits angerissen und betrifft Unterschiede von Geweben oder Substanzen hinsichtlich der Absorption von Strahlen verschiedener Energie. Bei der Knochendichtemessung mit zwei Energien (Dual Energy X-ray Absorptiometry, DEXA) wird sie seit mehr als 30 Jahren eingesetzt, seit mehr als 10 Jahren bei der DualEnergy-CT. Aktuelle Entwicklungen gehen dahin, die Absorption bei mehr als nur zwei Energien zu messen (Spektral$\mathrm{CT}$ ). Welche Vorteile dies über die DualEnergy-CT bietet, wird sich in den kommenden Jahren zeigen. Ungeheuer faszinierend ist ein Rückgriff auf den fast vergessenen Welle-Teilchen-Dualismus der Photonen, der in Form der Phasenkontrast- und Dunkelfeldbildgebung zu völlig neuen, bislang noch nicht bekannten Kontrasten führt [5].

Zur Zeit unseres Studiums arbeiteten Radiologen weitgehend im Dunkeln und deuteten schwarz-grau-weiße Rätselbilder - nicht sehr faszinierend. Die vergangenen, mehr als 30 Jahre haben uns eine Fülle von Bildern nie geahnter Plastizität, Genauigkeit, sogar Farbigkeit, vor allem aber Aussagekraft beschert. Es ist gut, dass Vieles hiervon gänzlich ohne Einsatz ionisierender Strahlung auskommt, deren Risiken auch im Bereich vergleichsweise niedriger Dosen in Studien z.B. an Arbeitern in der Nuklearindustrie inzwischen empirisch belegt sind [7]. Dennoch werden wir auch weiterhin nicht ohne ionisierende Strahlung auskommen, und solange die Prinzipien des Strahlenschutzes - Recht- fertigung, Begrenzung, Optimierung ernstgenommen und praktiziert werden, spricht nichts gegen ihre Anwendung. Die Stärken bildgebender Verfahren mit ionisierenden Strahlen wie Schnelligkeit, Spezifität oder Flexibilität sind dort eine Bereicherung, wo strahlungsfreie Verfahren ihre Schwächen haben, und es tut sich enorm viel - vielleicht nicht ganz so laut.

Ihre

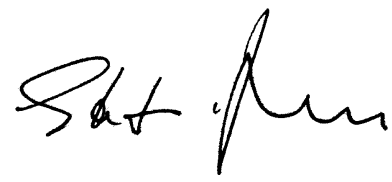

Prof. Dr. Stefan Delorme

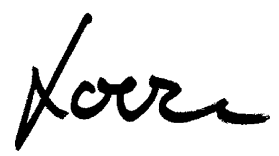

Prof. Dr. Reinhard Loose

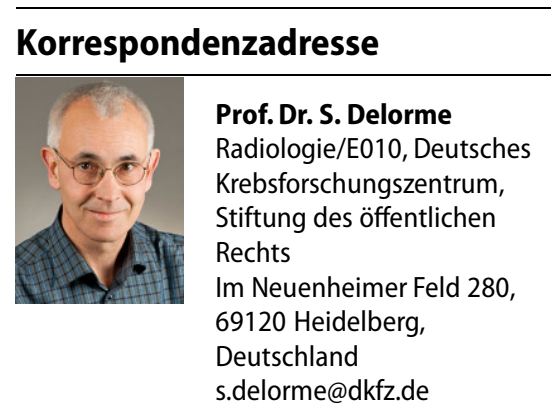

Interessenkonflikt. S. Delorme und R. Loose geben an, dass kein Interessenkonflikt besteht.

\section{Literatur}

1. Skornitzke S (2018) Iterative Verfahren zur Artefaktreduktion in der Computertomographie. Radiologe. https://doi.org/10.1007/s00117-0170327-7

2. Fiebich M, Weber D (2018) Digitale Volumentomographie. Radiologe. https://doi.org/10.1007/ s00117-018-0360-1

3. von Hardenberg J, Büsing K-A, Nuhn P, Ritter M (2018) Die Rolle des PSMA-PET/CT bei Patienten mit metastasiertem Prostatakarzinom. Radiologe. https://doi.org/10.1007/s00117-018-0358-8

4. Beuthien-Baumann B (2018) PET/MRT. Radiologe. https://doi.org/10.1007/s00117-018-0355-y

5. Pfeiffer F, Reiser M, Rummeny E (2018) Röntgen Phasenkontrast. Radiologe. https://doi.org/10. 1007/s00117-018-0357-9

6. DelormeS, Jenderka KV(2015) Diestille Revolution. Radiologe 55:935-936
7. Kreuzer M (2017) Krebsrisiko durch ionisierende Strahlung im Niedrigdosisbereich. Radiologe 57:517-518 